\title{
Multi-parameter optochemical sensing based on coated cascaded long-period fiber gratings and frequency division multiplexing
}

\author{
Qiuping Huang ${ }^{1}$, and Haiyun Chen ${ }^{2, *}$ \\ ${ }^{1}$ College of Mathematics, Physics and Information Engineering, Zhejiang Normal \\ University, 688 Yingbin Road, Jinhua, 321004, Zhejiang Province, People's \\ Republic of China, Tel: 0086-0579-82298249 \\ Email: hqp@zjnu.cn \\ ${ }^{2}$ Institute of Information Optics, Zhejiang Normal University, 688 Yingbin Road, \\ Jinhua, 321004, Zhejiang Province, People's Republic of China, \\ Tel: 0086-0579-82298863, Fax: 0086-0579-82298863 \\ Email: chy@zjnu.cn
}

\begin{abstract}
We propose a multi-parameter optochemical sensing technique based on coated cascaded long-period fiber gratings (CLPFG) and frequency division multiplexing (FDM). Three CLPFGs, each with identical period $\Lambda$ and length $L$ but different separation $\mathrm{S}$, are fabricated and connected with in-between fibers whose polymer jackets are not striped, which produces a composite spectrum that contains three fringe patterns with different frequencies. After a Fourier transform of the output spectrum, the characteristic peaks in Fourier spectrum are extracted with a filtering operation. The phases recovered by inverse transform of the respective filtered peaks allow the determination of the responses to respective measurands. A detailed investigation of sensing principle and methodology is developed. In experiment, the sensing system is composed of $\mathrm{CLPFG}_{1}\left(S_{1}=10 \mathrm{~mm}\right)$ coated with $\mathrm{TiO}_{2} / \mathrm{SnO}_{2}$ composite film, $\mathrm{CLPFG}_{2}\left(S_{2}=30 \mathrm{~mm}\right)$ coated with PAH/PAA film and bare $\mathrm{CLPFG}_{3}\left(S_{3}=80 \mathrm{~mm}\right)$, which act to measure relative humidity, $\mathrm{pH}$ value and concentration of $\mathrm{NaCl}$ solution, respectively. The results show that the responses to three parameters can be readily extracted and the sensitivities reach $0.025 \mathrm{rad} / \%, 0.687 \mathrm{rad} / \mathrm{pH}$ unit and $0.113 \mathrm{rad} / \%$, respectively.
\end{abstract}

Key words: Fiber optics, Optochemical sensing, Cascaded long-period fiber grating (CLPFG), Frequency division multiplexing (FDM)

\section{Introduction}

A long-period fiber grating (LPFG) fabricated by a periodic refractive index (RI) modulation in the fiber core with a typical period of few hundred micrometers couples the core mode to copropagating cladding modes, resulting in a set of discrete attenuation bands in the transmission spectrum [1]. By virtue of the advantages such as ease of fabrication, low

* Author to whom any correspondence should be addressed. 
insertion loss and back reflection, compactness and sensitiveness to the ambient physical parameters, LPFGs have been used in diverse sensing applications [2]. In recent years, great efforts have been dedicated to the research of LPFGs coated with sensitive films. The film RI or thickness will change by interacting with the measurands, which enables these coated LPFGs to act as chemo- [3] and bio-sensors [4]. In addition, the presence of film with proper parameters improves the sensitivity in a great manner via cladding mode reorganization mechanism [5]. Cascaded long-period fiber gratings (CLPFG) consist of two identical 3-dB LPFGs with a separation fiber in between, which forms an in-fiber Mach-Zenhder interferometer (MZI) whose transmission spectrum is characterized as sinusoidal channeled patterns [6]. In addition to the sensing mechanism of LPFGs, the separation fiber introduces a phase difference between core and cladding modes, which leads to higher sensitivity of CLPFG than that is based on a single LPFG. Furthermore, the fringe frequency is dominantly dependant on the separation S [7], which offers potential applications of CLPFG-based MZIs in frequency division multiplexing (FDM).

Multi-parameter sensing is another focus in LPFG-based sensing field. Among various established multi-parameter sensing techniques, a differential modulation method is commonly exploited to determine the responses to different parameters [8,9]. However, this method is usually limited in two-parameter sensing and not suitable for multipoint sensing since only an integrated LPFG-based device is involved. In order to overcome this disadvantage, a multiplexing technique is necessary for multipoint sensing applications. Murphy R P et al. demonstrated the multiplexing of a serial array of LPFG-based MZIs by the application of Fourier transform technique to the transmission spectrum [10], which provided a methodological foundation for frequency division multiplexing of CLPFG-based sensors. However, a detailed Fourier processing of the composite MZI fringes and the applications of this multiplexing technique in multi-parameter optochemical sensing based on coated CLPFG are not been reported. In fact, multi-parameter optochemical sensing is very important in numerical areas such as production safety, environment monitoring and food industry where diverse chemical parameters ( $\mathrm{pH}$, humidity, solution concentration, etc.) need to be measured.

In this work for the first time, to the best of our knowledge, we demonstrated a multi-parameter optochemical sensing technique based on coated CLPFG and FDM. Three CLPFGs with different separations are fabricated for three measurands. The CLPFGs are connected with two in-between fibers whose polymer jackets are not stripped so as to eliminate the cladding-mode crosstalk between two CLPFGs. As a result, the output spectrum is a simple product of the three respective normalized spectra associated with the three CLPFGs. Followed by a phase recovery, a Fourier transform of the output spectrum and filtering in the frequency domain are performed, which allows the extraction and determination of the responses to the respective measurands. In experiment, the sensing system is composed of CLPFG $\left(S_{1}=10 \mathrm{~mm}\right)$ coated with $\mathrm{TiO}_{2} / \mathrm{SnO}_{2}$ composite film, CLPFG $\left(S_{2}=30 \mathrm{~mm}\right)$ coated with PAH/PAA film and bare $\mathrm{CLPFG}_{3}\left(S_{3}=80 \mathrm{~mm}\right)$, which act to measure relative humidity $(\mathrm{RH}), \mathrm{pH}$ value and $\mathrm{NaCl}$ solution concentration (SC), respectively. The results show that the responses to $\mathrm{RH}, \mathrm{pH}$ and $\mathrm{SC}$ can be readily distinguished and the sensitivities are $0.025 \mathrm{rad} / \%, 0.687 \mathrm{rad} / \mathrm{pH}$ unit and $0.113 \mathrm{rad} / \%$, respectively.

\section{Principle and methodology}


The schematic diagram of a tri-parameter optochemical sensing system is illustrated in figure 1(a), where a coated/bare CLPFG serves as a sensing element. The polymer jacket of connecting fiber between two CLPFGs are remained, which implies that the residual cladding modes after a CLPFG will be exhausted due to the absorption of polymer and thus, are not capable to reach the next CLPFG. Therefore, the output spectrum after the whole system is simply the product of the three respective normalized spectra associated with three elements. In other words, the length of connecting fiber exerts little influence on the output spectrum, which is of spectial importance for multipoint sensing since the length of in-between fiber can be taken as needed. Three CLPFGs with identical periods $\Lambda$ and lengths $L$ but different separations $S$ are inscribed in single-mode fibers. CLPFG 1 coated with $\mathrm{TiO}_{2} / \mathrm{SnO}_{2}$ composite film, $\mathrm{CLPFG}_{2}$ with $\mathrm{PAH} / \mathrm{PAA}$ film and bare $\mathrm{CLPFG}_{3}$ act to measure $\mathrm{RH}, \mathrm{pH}$ and $\mathrm{SC}$, respectively. Therefore, the output composite spectrum contains three fringe patterns with different frequencies induced by the different separations in CLPFGs [10]. Taking coated $\mathrm{CLPFG}_{2}$ as an example, the sensing principle and signal processing method are to be elucidated.

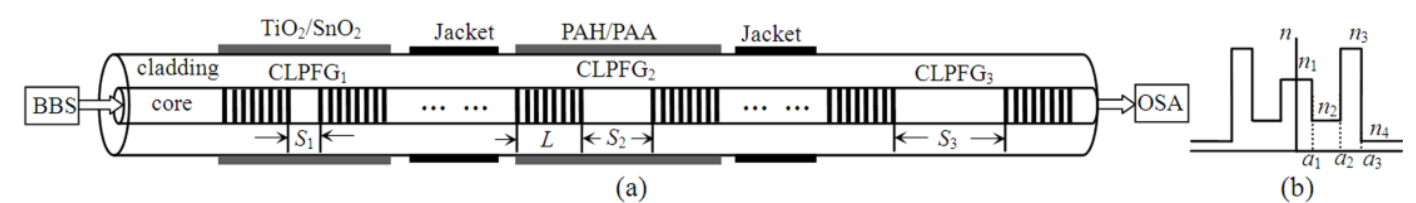

Figure 1. Schematic of tri-parameter optochemical sensing system: (a) structural diagram; (b) refractive index profile of a coated CLPFG.

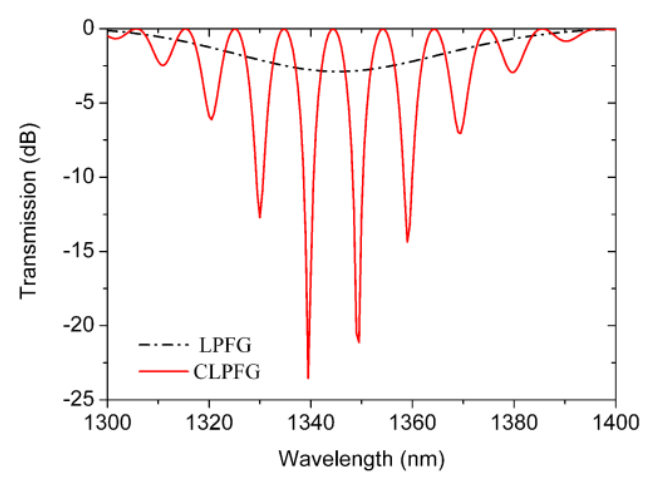

Figure 2. Simulated transmission spectrum of a coated CLPFG.

The refractive index profile of a coated CLPFG is shown in figure 1(b), where $n_{3}$ denotes the film RI, while the film thickness can be given as $h_{3}=a_{3}-a_{2}$. Based on coupled-mode theory and transfer matrix method [11], the transmission spectrum of coated $\mathrm{CLPFG}_{2}$ is calculated and shown in figure 2, where $n_{3}=1.55, h_{3}=420 \mathrm{~nm}, \Lambda=300 \mu \mathrm{m}, L=6 \mathrm{~mm}$ and $S=30 \mathrm{~mm}$. It is evident that the spectrum manifests itself as a sinusoidal channeled pattern within the envelope of an attenuation band associated with a single LPFG. This fringe pattern is a result of MZI where the separation fiber introduces a phase difference between the core and cladding modes [6]. A variation of measurand causes a change of film RI via the interaction between film and measurand, which leads to a change of phase difference in addition to a shift of the envelope. Thus, a CLPFG-based film sensor possesses a higher sensitivity than that based on a single LPFG [12]. 

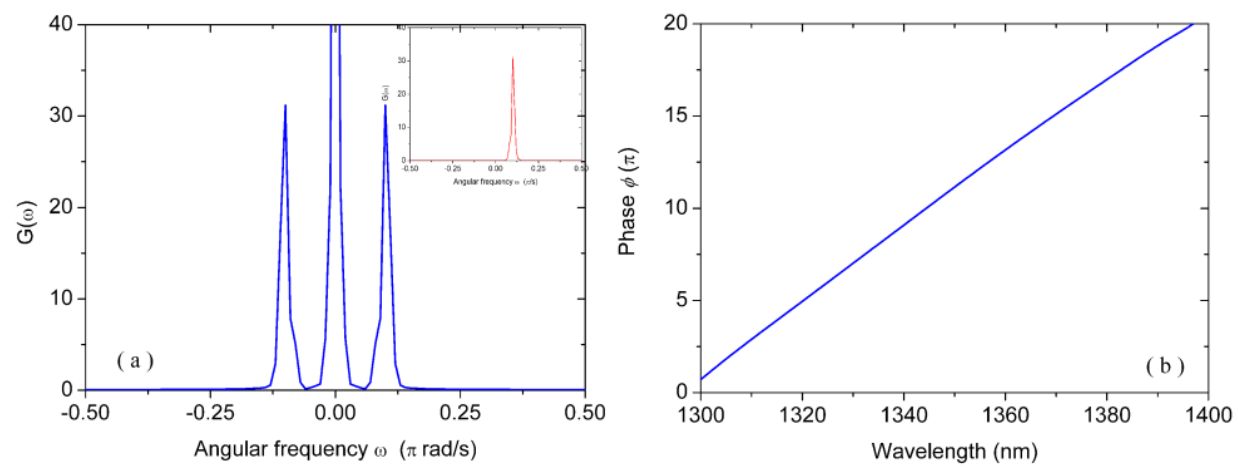

Figure 3. Fourier processing of fringe pattern: (a) Fourier spectrum (inset: filtered spectrum); (b) unwrapped phase.

Fourier-transform method is a powerful tool to process this kind of periodically modulated spectral signals [13]. A generalized expression for MZI fringe produced by a CLPFG can be given as:

$$
g(\lambda)=a(\lambda)+b(\lambda) \times \cos [\phi(\lambda)]
$$

where $\phi(\lambda)$ is the phase difference between two modes. Eq. (1) can be further rewritten as:

$$
g(\lambda)=a(\lambda)+c(\lambda)+c^{*}(\lambda)
$$

where

$$
c(\lambda)=\frac{1}{2} b(\lambda) \exp [j \phi(\lambda)]
$$

with $j=\sqrt{-1}$ and $*$ denoting complex conjugate. Eq. (2) is Fourier transformed with respect to $\lambda$ by the use of a fast-Fourier transform (FFT) algorithm, which gives:

$$
G(\omega)=A(\omega)+C(\omega)+C^{*}(\omega)
$$

where the capital letters denote the Fourier spectra and $\omega$ is the angular frequency in wavelength domain. Illustrated in figure 3(a) is the Fourier spectrum of the fringe pattern shown in figure 2, which shows that the two peaks corresponding to $\mathrm{C}$ and $\mathrm{C}^{*}$ are separated by the broadened zero peak and placed symmetrically to the origin. We can make use either of the two peaks, say C, by filtering out the $\mathrm{C}^{*}$ as well as the zero peak, which is shown in the inset of Fig. 3(a). This remaining spectrum is no longer symmetrical; thus it yields a nonzero imaginary part in $c(\lambda)$ after inverse Fourier transform. Then, we take the $\log$ (base $e$ ) of Eq. (3):

$$
\ln [c(\lambda)]=\ln \left[\frac{1}{2} b(\lambda)\right]+j \phi(\lambda)
$$

Now we can recover the phase $\phi(\lambda)$ in the imaginary part in Eq. (5). It is stressed that the phase so obtained is indeterminate to a factor of $2 \pi$ and, in most cases, a computer-generated function subroutine gives a principal value form $-\pi$ to $\pi$. A unwrapping algorithm [14] is necessary and effective to correct these discontinuities. figure 3(b) shows that the unwrapped phase increases continually and monotonically with wavelength. For a coated CLPFG, the interaction between sensitive film and environment induces a change of the film RI, which gives rise to the change of phase. The dependence of phase at $1345.0 \mathrm{~nm}$ on film RI is illustrated in figure 4, which exhibits 
an approximately linear relation. A linear fitting shows that the sensitivity reaches $706.42 \mathrm{rad} / \mathrm{RIU}$. Therefore, we can determine the change of measurand by interrogating the recovered phase at a fixed wavelength.

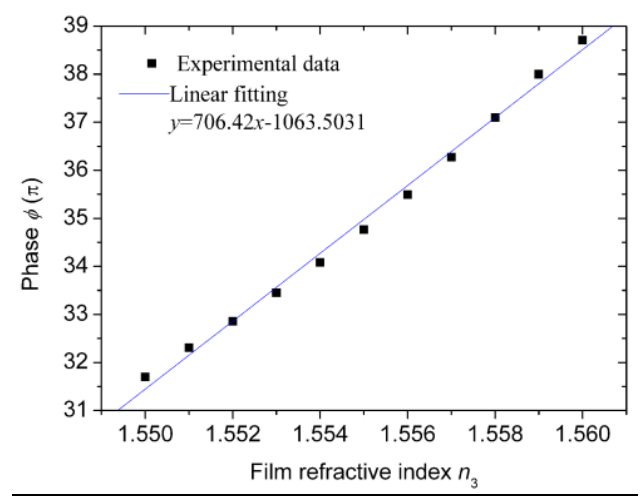

Figure 4. Dependence of the phase on film refractive index $(\lambda=1345.0 \mathrm{~nm})$.

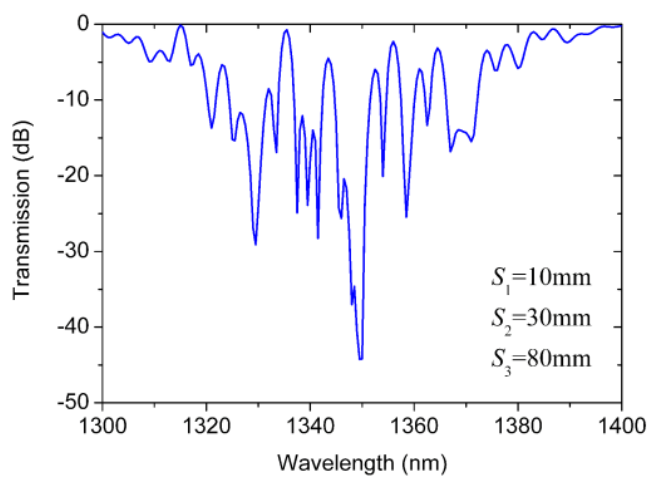

Figure 5. Composite transmission spectrum of three CLPFGs with different separations.
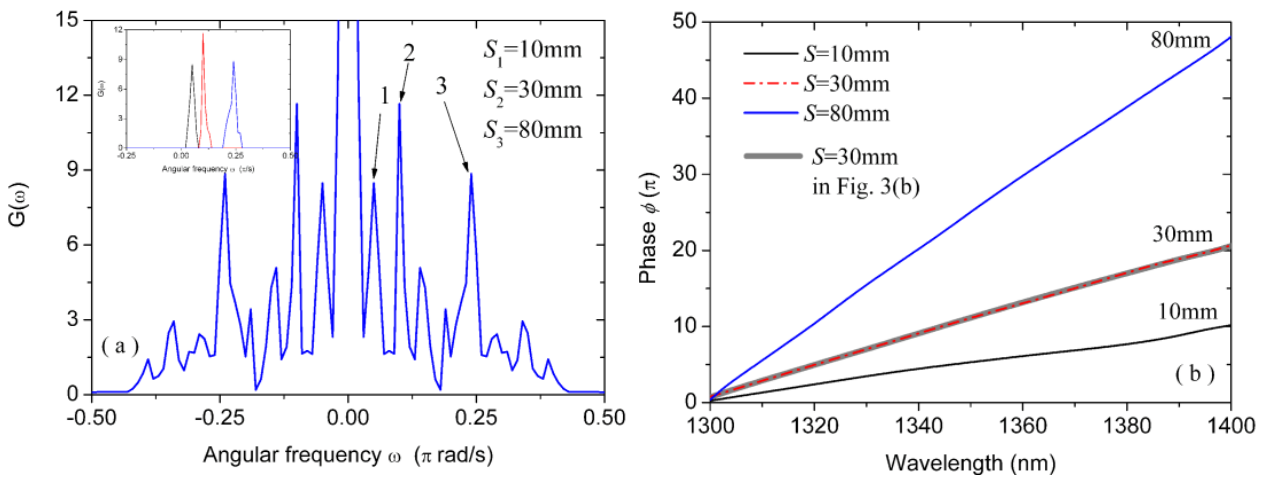

Figure 6. Fourier processing of composite fringe patterns: (a) Fourier spectrum (inset: three filtered characteristic peaks); (b) unwrapped phase.

It has been revealed that the frequency of interference fringe produced by a CLPFG is approximately linear to the separation [7]. When several CLPFGs with different separations are connected, as shown in figure 1, the output composite spectrum contains various fringe patterns with different frequencies. Shown in figure 5 is the composite spectrum which is produced by three CLPFGs with separations being $10 \mathrm{~mm}, 30 \mathrm{~mm}$ and $80 \mathrm{~mm}$. The corresponding Fourier spectrum is illustrated in figure 6(a), where the number $(1,2,3)$ marked peaks are the three characteristic peaks associated with three separations $10 \mathrm{~mm}, 30$ $\mathrm{mm}$ and $80 \mathrm{~nm}$, respectively. The sum and difference frequencies are also present. For this kind of FDM-based multi-parameter sensing system, it is important to ensure that the 
frequency components do not overlap by selecting proper separations. Moreover, the separation imbalances must be chosen to ensure that the sum and difference frequency components present in the Fourier spectrum are not equal to the characteristic frequencies. The filtered characteristic frequencies are shown in the inset of figure 6(a) and the corresponding recovered phases are illustrated in figure 6(b). It is evident that the recovered phase for $S=30 \mathrm{~mm}$ from the composite Fourier spectrum is almost identical to that in figure 3(b), which implies that the filtered characteristic frequencies carry sufficient information to represent the respective fringe characteristics. Therefore, this multiplexing technique based on CLPFG and FDM is feasible and effective.

\section{Fabrication}

In experiment, the used fiber was Corning SMF-28 which was hydrogen loaded in advance for a week to enhance its photosensitivity. The gratings were fabricated by illuminating the fiber core with $248 \mathrm{~nm} \mathrm{KrF}$ excimer laser. The pulse energy, frequency and exposure time are $24 \mathrm{~mJ}, 150 \mathrm{~Hz}$ and 13s, respectively. The period and length of a single LPFG is $300 \mu \mathrm{m}$ and $14.4 \mathrm{~mm}$, respectively. In this case, the peak loss is approximately $3 \mathrm{~dB}$ so as to obtain the optimum fringe contrast for CLPFG. Then, the fiber is moved along axis direction with a certain distance, which is followed by the inscription of the other identical LPFG. Thus, a CLPFG is formed and the motion distance is very the separation. The separations of three fabricated CLPFGs are: $S_{1}=10 \mathrm{~mm}, S_{2}=30 \mathrm{~mm}$ and $S_{3}=80 \mathrm{~mm}$.

Aiming to implement optochemical sensing, CLPFG ${ }_{1}$ is coated with $\mathrm{TiO}_{2} / \mathrm{SnO}_{2}$ composite film for RH sensing and $\mathrm{CLPFG}_{2}$ is coated with $\mathrm{PAH} / \mathrm{PAA}$ film for $\mathrm{pH}$ sensing, while the bare $\mathrm{CLPFG}_{3}$ is directly applied for SC sensing. Three CLPFGs are connected with in-between fibers using a fusion splicer (FITEL, S178). The deposition and characterization of sol-gel derived $\mathrm{TiO}_{2} / \mathrm{SnO}_{2}$ composite film have been reported in our previous work [15]. The film refractive index and thickness were measured to be 2.3789 and $72.9 \mathrm{~nm}$, respectively, which fall in the mode-transition region with high sensitivity according to the design methodology for coated LPFGs[18].

Now, we take $\mathrm{CLPFG}_{2}$ as an example to demonstrate the deposition of PAH/PAA film and investigate the influence of film deposition on the output fringe. The $\mathrm{pH}$-sensitive film is fabricated by a self-assembly method [16], where poly(allylamine hydrochloride) (PAH) (Mw $\sim 15000$ ) and poly(acrylic acid) (PAA) ( $\mathrm{Mw} 45000$ ) serve as polycation and polyanion, respectively. Aqueous solutions of the polymers $(2 \mathrm{~g} / \mathrm{L})$ were prepared using deionized water

$(18 \mathrm{M} \Omega \cdot \mathrm{cm})$ and adjusted to $\mathrm{pH}=4.5$. The whole $\mathrm{CLPFG}_{2}$ including two $\mathrm{LPFG}$ and separation fiber was first immersed into $\mathrm{KOH}$ solution $(1 \mathrm{Mol} / \mathrm{L})$ for $20 \mathrm{~min}$ in order to acquire negative charge and then was cleaned with deionized water and dried with nitrogen gas. The fiber was then immersed into PAH and PAA solutions alternatively with reaction of 4 min to form a monolayer of polymer through electrostatic adsorption. Each immersion operation was followed by a rigorous rinse in deionized water in order to remove the excess of material adsorbed. Each polycation/polyanion layer (PAH/PAA) is called a bilayer. This process was repeated up to 38 bilayers and then $\mathrm{CLPFG}_{2}$ was baked in an oven at $60{ }^{\circ} \mathrm{C}$ for $60 \mathrm{~min}$.

The refractive index and thickness of fabricated PAH/PAA film are measured to be 1.55 and $304 \mathrm{~nm}$, respectively. The transmission spectra of $\mathrm{CLPFG}_{2}$ before and after the film 
deposition are shown in figure 7. A comparison between these two spectra shows that the fringe pattern shifts towards shorter wavelength. This is dependent on the fact that the deposition of film increases the effective refractive index of cladding mode and thus, results in the decrease of resonant wavelength according to the phase-matching condition of LPFG [11]. Another conspicuous change is the decrease of fringe contrast, which mainly stems from the influence of film on the coupling strength between the core and cladding mode. The peak loss of a single LPFG in fabrication is controlled to be $3 \mathrm{~dB}$; thus, the fringe contrast of bare CLPFG reaches maximum. The presence of sensitive film changes the coupling strength, which causes the peak loss of a single LPFG to deviate from $3 \mathrm{~dB}$ and thus, results in the great degradation of the fringe contrast. Another factor causing the contrast degradation is the wave absorption by the film, which is attributed to the nonzero imaginary part of the film RI [17]. As a result, the fringe contrast will deteriorate with further film deposition, which is the reason why the thickness $304 \mathrm{~nm}$ is adopted. It is stressed that the thickness $304 \mathrm{~nm}$ as well as RI 1.55 are not in, but near to the mode-transition region for coated LPFGs, which means that a relatively high sensitivity is still available. The other less evident change is the slight decrease of the fringe frequency, which is attributed to the decrease of phase difference between the core and cladding mode caused by the film deposition since the film covers the separation fiber.

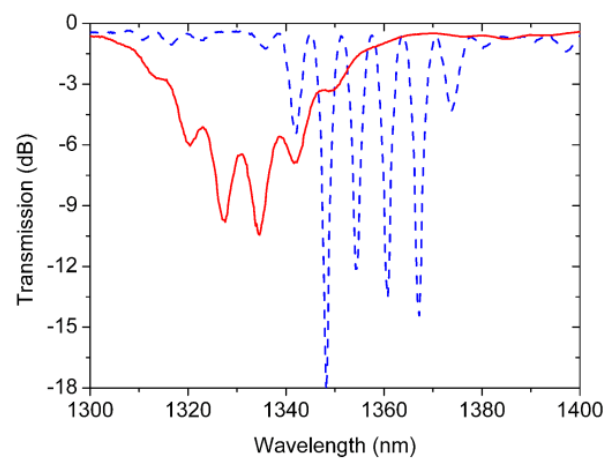

Figure 7. Transmission spectrum of $\mathrm{CLPFG}_{2}$ before (dashed line) and after (solid line) deposition of PAH/PAA film

\section{Results}

The sensing test of this fabricated tri-parameter optochemical sensing system was carried out. As shown in figure 1(a), light from broadband source (BBS) was injected into $\mathrm{CLPFG}_{1}$ and the composite spectrum outputted from $\mathrm{CLPFG}_{3}$ was monitored and recorded by an optical spectrum analyzer (OSA, YOKOGAWA, AQ6370). Each sensing element was straightly fixed with a U-shaped frame so as to eliminate the response to strain and curvature. The test was performed at an environmental temperature $20 \pm 0.5^{\circ} \mathrm{C}$, which minimizes the interference of temperature fluctuation. The coated $\mathrm{CLPFG}_{2}$ and bare $\mathrm{CLPFG}_{3}$ were immersed into respective aqueous solutions, whereas the coated $\mathrm{CLPFG}_{1}$ was directly exposed to moist air. Therefore, the cross response among these three parameters is negligible. The output transmission spectrum for $\mathrm{RH}=53 \%, \mathrm{pH}=7$ and $\mathrm{SC}=0 \%$ is shown in figure $8(\mathrm{a})$, which seems quite different from the simulated results in figure 5. This arises mainly from the fact that the realistic structural parameters in experiments may deviate from that in simulation. The film deposition $\left(\mathrm{CLPFG}_{1}\right.$ and $\left.\mathrm{CLPFG}_{2}\right)$ and different surrounding refractive indices cause the three respective fringe envelope peaks to locate at different wavelengths, although the periods of 
fabricated CLPFGs are identical. Nevertheless, this exerts little influence on the measurements, since the fringe frequencies and phases are interrogated in this FDM system. The Fourier spectrum is illustrated in figure 8(b). Evidently, there are three distinguishable characteristic peaks which are filtered and shown in the inset. The recovered phases are shown in figure 8(c), which quantitatively represent the measurands and thus, the variation of each parameter can be determined by interrogating the respective phase information.
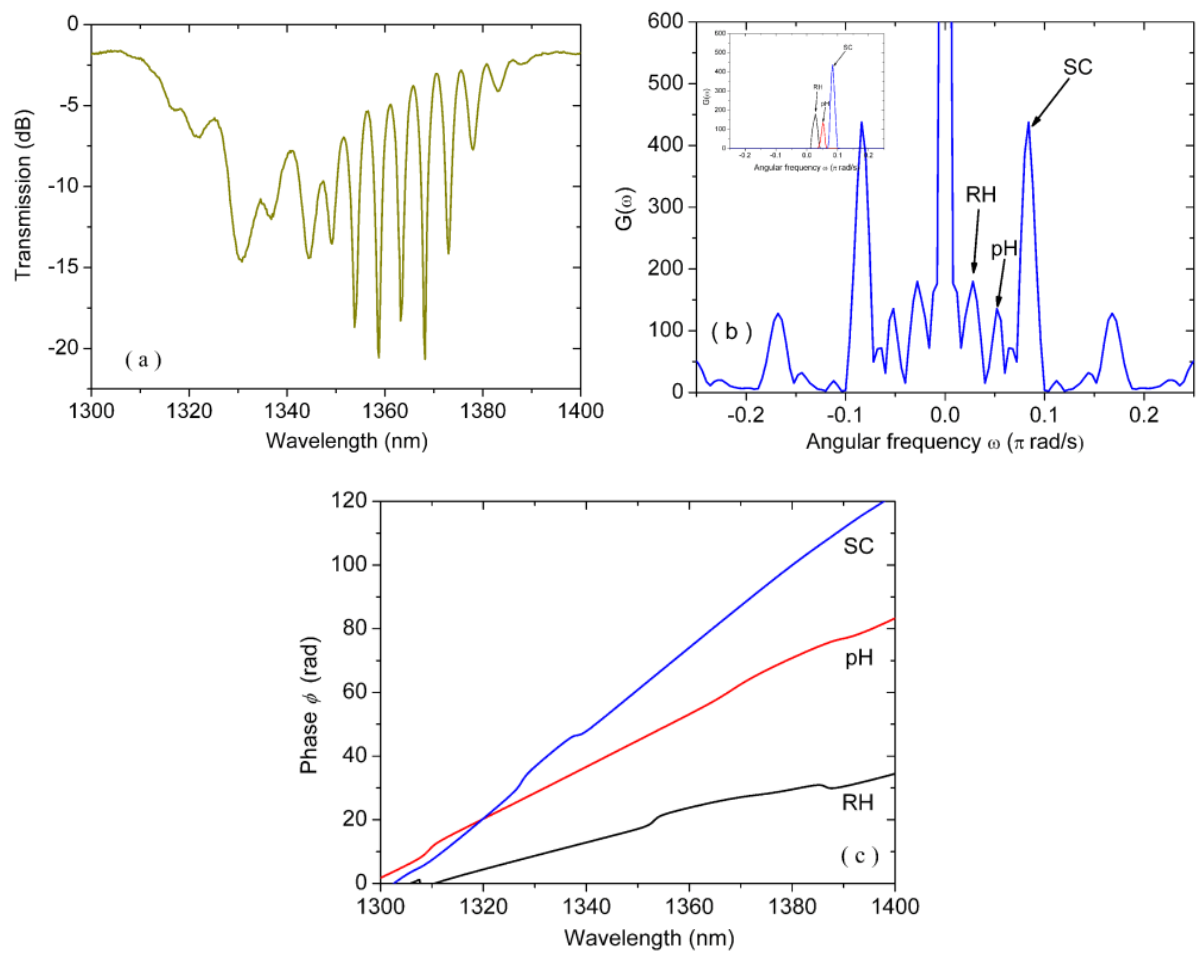

Figure 8. Fourier processing of measured composite spectrum $(\mathrm{RH}=53 \%, \mathrm{pH}=7, \mathrm{SC}=0 \%)$ : (a) composite transmission spectrum; (b) Fourier spectrum (inset: filtered spectra); (c) recovered phases.

The response of CLPFG 1 coated with $\mathrm{TiO}_{2} / \mathrm{SnO}_{2}$ film to $\mathrm{RH}$ varying from $53 \%$ to $82 \%$ is shown in figure 9, which exhibits an approximately linear dependence of phase on RH. The linear fitting results show that the sensitivity of this sensing element reaches $0.025 \mathrm{rad} / \%$. A comparison between the results for increasing and decreasing $\mathrm{RH}$ manifests excellent reversibility and stability. The sensing mechanism of $\mathrm{TiO}_{2} / \mathrm{SnO}_{2}$ film coated CLPFG relies on the high adsorption capability of water molecular of the porous film [15]. The $\mathrm{pH}$ sensing element $\left(\mathrm{CLPFG}_{2}\right.$ coated with PAH/PAA film) was tested with $\mathrm{HCl}$ and $\mathrm{NaOH}$ aqueous solutions; the results are illustrated in figure 10. One can see that the phase decreases with increasing of $\mathrm{pH}$ value in acidic range, and also decreases with decreasing of $\mathrm{pH}$ value in alkaline range. This is dependent on the fact that the ionization state of the PAH/PAA multi-layer is modulated by the $\mathrm{pH}$ value through swelling/deswelling and reaches maximum at the neutral $\mathrm{pH}$ [17]. The sensitivities of $\mathrm{pH}$-sensing element are $-0.687 \mathrm{rad} / \mathrm{pH}$ unit and $0.649 \mathrm{rad} / \mathrm{pH}$ unit for acidic and alkaline solutions, respectively. $\mathrm{NaCl}$ solutions with different concentrations are employed for concentration sensing tests of $\mathrm{CLPFG}_{3}$. The solution RI is linearly dependent on the concentration and the measurement of solution RI with Abbe refractometer reveals that the linear relation can be described as $y=0.00184 x+1.3333$. The sensing results shown in figure 11 exhibit a reversible linear response and the sensitivity is 
circa $0.113 \mathrm{rad} / \%$. Different parameter combinations and the corresponding recovered phases are listed in table 1, which confirms that the recovered phase via filtering and reverse Fourier transform effectively characterize the parameter variations. The data illustrated in table 1 also show that there is a spectral crosstalk among three parameter responses. Nevertheless, we can see that the crosstalk is in the order of $10^{-3} \mathrm{rad}$, which is in an acceptable range comparing to the sensitivities.

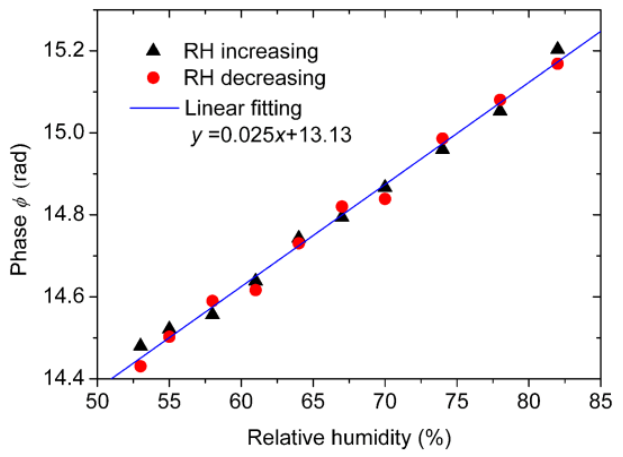

Figure 9. Response of $\mathrm{CLPFG}_{1}$ coated with $\mathrm{TiO}_{2} / \mathrm{SnO}_{2}$ film to $\mathrm{RH}(\lambda=1329.0 \mathrm{~nm}, \mathrm{pH}=7$, $\mathrm{SC}=0 \%)$.
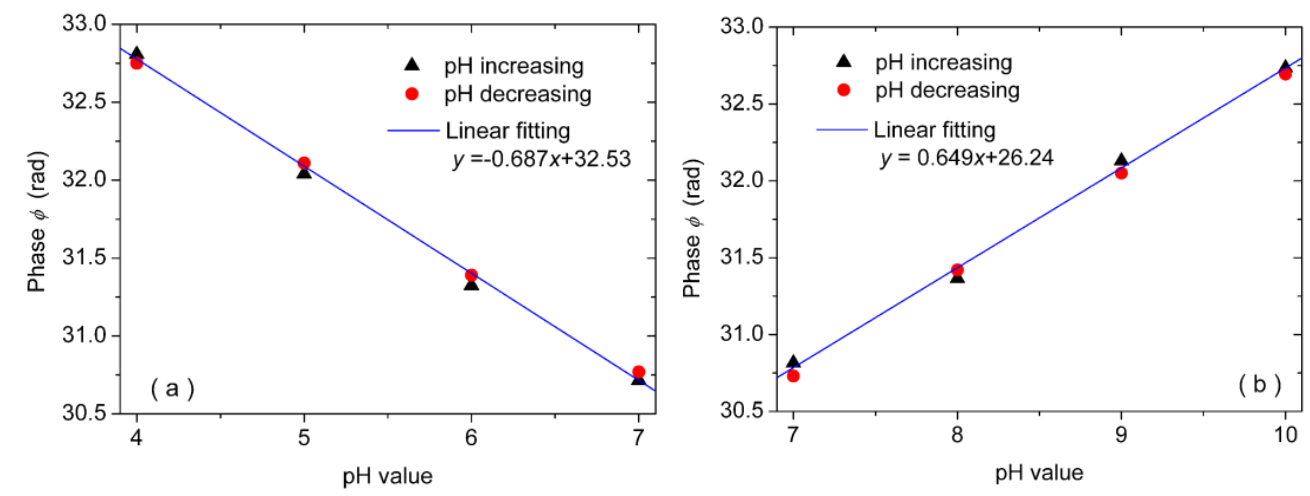

Figure 10. Response of $\mathrm{CLPFG}_{2}$ coated with $\mathrm{PAH} / \mathrm{PAA}$ film to $\mathrm{pH}$ value in (a) acidic and (b) alkaline solutions $(\lambda=1332.6 \mathrm{~nm}, \mathrm{RH}=53 \%, \mathrm{SC}=0 \%)$.

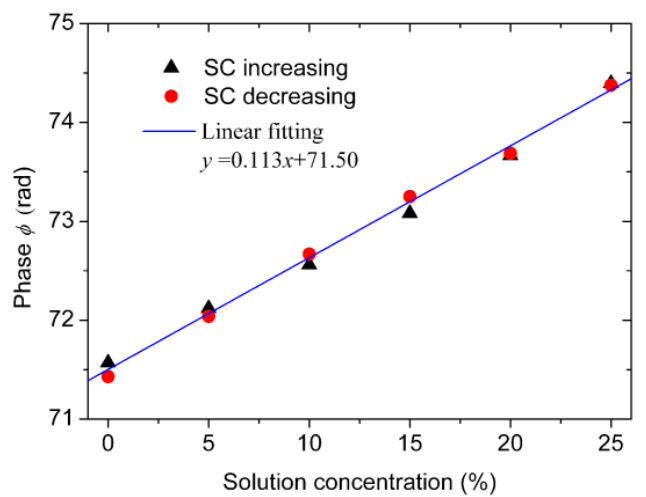

Figure 11. Response of $\mathrm{CLPFG}_{3}$ to $\mathrm{NaCl}$ solution concentration $(\lambda=1358.2 \mathrm{~nm}, \mathrm{RH}=53 \%, \mathrm{pH}=7)$.

Table 1. Different parameter combinations and the corresponding recovered phases

\begin{tabular}{cccccc}
\hline $\mathrm{RH}(\%)$ & $\mathrm{pH}$ & $\mathrm{SC}(\%)$ & $\phi_{\mathrm{RH}}(\mathrm{rad})$ & $\phi_{\mathrm{pH}}(\mathrm{rad})$ & $\phi_{\mathrm{SC}}(\mathrm{rad})$ \\
\hline 53 & 7 & 0 & 14.5436 & 30.5398 & 71.6710 \\
53 & 10 & 0 & 14.5432 & 40.9438 & 71.6712 \\
74 & 7 & 0 & 15.1646 & 30.5382 & 71.6711
\end{tabular}




\begin{tabular}{llllll}
53 & 7 & 10 & 14.5430 & 30.5379 & 72.5925 \\
64 & 8 & 15 & 14.7456 & 31.0243 & 73.0982 \\
\hline
\end{tabular}

\section{Conclusions}

In summary, we have demonstrated a multi-parameter optochemical sensing technique based on coated CLPFGs and FDM. CLPFGs with different separations produce fringe patterns with different frequencies and are contained in the output transmission spectrum. A Fourier transform of the output spectrum allows the identification of the characteristic peaks which can be extracted by a bandpass filtering operation. Therefore, the measurands are determined by interrogating the recovered phases. In experiment, a tri-parameter optochemical sensing system is set up, which consisted of $\mathrm{CLPFG}_{1}(S=10 \mathrm{~mm})$ coated with $\mathrm{TiO}_{2} / \mathrm{SnO}_{2}$ composite film, $\mathrm{CLPFG}_{2}(S=30 \mathrm{~mm})$ coated with PAH/PAA film and bare $\mathrm{CLPFG}_{3}(S=80 \mathrm{~mm})$ sensing for $\mathrm{RH}, \mathrm{pH}$ and $\mathrm{SC}$, respectively. The testing results show that the responses to $\mathrm{RH}, \mathrm{pH}$ and $\mathrm{SC}$ can be readily distinguished and extracted. The sensitivities are $0.025 \mathrm{rad} / \%, 0.649 \mathrm{rad} / \mathrm{pH}$ unit and $0.113 \mathrm{rad} / \%$, respectively, with excellent reversibility and stability. It is stressed that temperature interference is the intrinsic limitation for LPFG-based sensors. A thermo test evidenced a red shift of $0.3 \mathrm{~nm}$ for temperature from $20^{\circ} \mathrm{C}$ to $25^{\circ} \mathrm{C}$. The compensation of temperature crosstalk is the further purchase in addition to the current work for practical applications.

\section{Acknowledgement}

This work is jointly supported by the National Science Foundation of China (51302248), Research program of Education Department of Zhejiang Province (Y201534206), the Zhejiang Normal University Foundation for Doctor (ZC304014028) and the Key Laboratory of Optical Information Detection and Display Technique in Zhejiang Province.

\section{Reference}

[1] Vengsarkar A M, Lemaire P J, Judkins J B, Bhatia V, Erdogan T and Sipe J E 1996 Long-period fiber gratings as band-rejection filters J. lightwave technol. 14 58-64

[2] James S W and Tatam R P 2003 Optical fibre long-period grating sensors: characteristics and applications Meas. Sci. Technol. 15 R49-61

[3] Cusano A, Iadicicco A, Pilla P, Contessa L, Campopiano S, Cutolo A, Giordano M and Guerra M 2006 Coated long-period fiber gratings as high-sensitivity optochemical sensors J. Lightw. Technol. 24 1776-86.

[4] Smietana M, Bock W J, Mikulic P, Ng A, Chinnappan R and Zourob M 2011 Detection of bacteria using bacteriophages as recognition elements immobilized on long-period fiber gratings Opt. Express $197971-78$.

[5] Cusano A, Iadicicco A, Pilla P, Contessa L, Campopiano S and Cutolo A 2005 Cladding mode reorganization in high-refractive-index-coated long-period grating: effects on the refractive-index sensitivty Opt. Lett. 30 2536-38.

[6] Lee B H and Nishii J 1998 Bending sensitivity of in-series long-period fiber gratings Opt. Lett. 23 1624-26.

[7] Lee B H and Nishii J 1999 Dependence of fringe spacing on the grating separation in a 
long-period fiber grating pair Appl. Opt. 36 3450-59.

[8] Bhatia V, Campbell D, Claus R O and Vengsarkar A M 1997 Simultaneous strain and temperature measurement with long-period gratings Opt. Lett. 22 648-50.

[9] Rego G, Falate R, Ivanov O and Santos J L 2007 Simultaneous temperature and strain measurements performed by a step-changed arc-induced long-period grating Appl. Opt. 46 1392-96.

[10] Murphy R P, James S W and Tatam R P 2007 Multiplexing of fiber-optic long-period grating-based interferometric sensors J. Lightw. Technol. 25 825-29.

[11] Erdogan T 1997 Fiber grating spectra J. lightwave technol. 15 1277-94

[12] James S W, Ishaq I, Ashwell G J and Tatam R P 2005 Cascaded long-period gratings with nanostructured coatings Opt. Lett. 30 2197-99.

[13] Takeda M, Ina H and Kobayashi S 1982 Fourier-transform method of fringe-pattern analysis for computer-based topography and interferometry J. Opt. Soc. Am. 72 156-60.

[14] Kreis T 1986 Digital holographic interference-phase measurement using the Fourier-transform method J. Opt. Soc. Am. A 3 847-55.

[15] Chen H Y, Gu Z T and Gao K 2014 Humidity sensor based on cascaded chirped long-period fiber gratings coated with $\mathrm{TiO}_{2} / \mathrm{SnO}_{2}$ composite films Sens. Actuators B: Chem. 196 18-22.

[16] Villar I D, Achaerandio M, Matias I R and Arregui F J 2005 Deposition of overlays by electrostatic self-assembly in long-period fiber gratings Opt. Lett. 30 720-22.

[17] Corres J M, Villar I D, Matias I R and Arregui F J 2007 Fiber-optic pH-sensor in long-period fiber gratings using electrostatic self-assembly Opt. Lett. 32 29-31.

[18] Pilla P, Trono C, Baldini F, Chiavaioli F, Giordano M and Cusano A 2012 Giant sensitivity of long period gratings in transition mode near the dispersion turning point: an integrated design approach Opt. Lett. 37 4152-54.

\section{Figure captions}

Figure 1. Schematic of tri-parameter optochemical sensing system: (a) structural diagram; (b) refractive index profile of a coated CLPFG.

Figure 2. Simulated transmission spectrum of a coated CLPFG.

Figure 3. Fourier processing of fringe pattern: (a) Fourier spectrum (inset: filtered spectrum); (b) unwrapped phase.

Figure 4. Dependence of the phase on film refractive index $(\lambda=1345.0 \mathrm{~nm})$.

Figure 5. Composite transmission spectrum of three CLPFGs with different separations.

Figure 6. Fourier processing of composite fringe patterns: (a) Fourier spectrum (inset: three filtered characteristic peaks); (b) unwrapped phase.

Figure 7. Transmission spectrum of $\mathrm{CLPFG}_{2}$ before (dashed line) and after (solid line) deposition of PAH/PAA film

Figure 8. Fourier processing of measured composite spectrum ( $\mathrm{RH}=53 \%, \mathrm{pH}=7, \mathrm{SC}=0 \%$ ): (a) composite transmission spectrum; (b) Fourier spectrum (inset: filtered spectra); (c) recovered phases.

Figure 9. Response of $\mathrm{CLPFG}_{1}$ coated with $\mathrm{TiO}_{2} / \mathrm{SnO}_{2}$ film to $\mathrm{RH}(\lambda=1329.0 \mathrm{~nm}, \mathrm{pH}=7$, $\mathrm{SC}=0 \%)$. 
Figure 10. Response of $\mathrm{CLPFG}_{2}$ coated with $\mathrm{PAH} / \mathrm{PAA}$ film to $\mathrm{pH}$ value in (a) acidic and (b) alkaline solutions $(\lambda=1332.6 \mathrm{~nm}, \mathrm{RH}=53 \%, \mathrm{SC}=0 \%)$.

Figure 11. Response of $\mathrm{CLPFG}_{3}$ to $\mathrm{NaCl}$ solution concentration ( $\left.\lambda=1358.2 \mathrm{~nm}, \mathrm{RH}=53 \%, \mathrm{pH}=7\right)$.

\section{Table caption}

Table 1 Different parameter combinations and the corresponding recovered phases

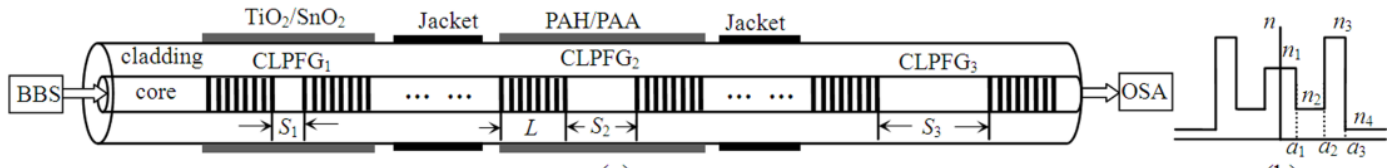

(a)

(b)

Figure 1. Schematic of tri-parameter optochemical sensing system: (a) structural diagram; (b) refractive index profile of a coated CLPFG.

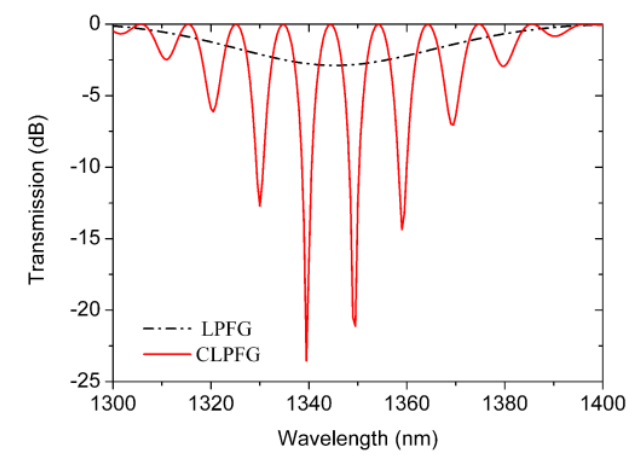

Figure 2. Simulated transmission spectrum of a coated CLPFG.
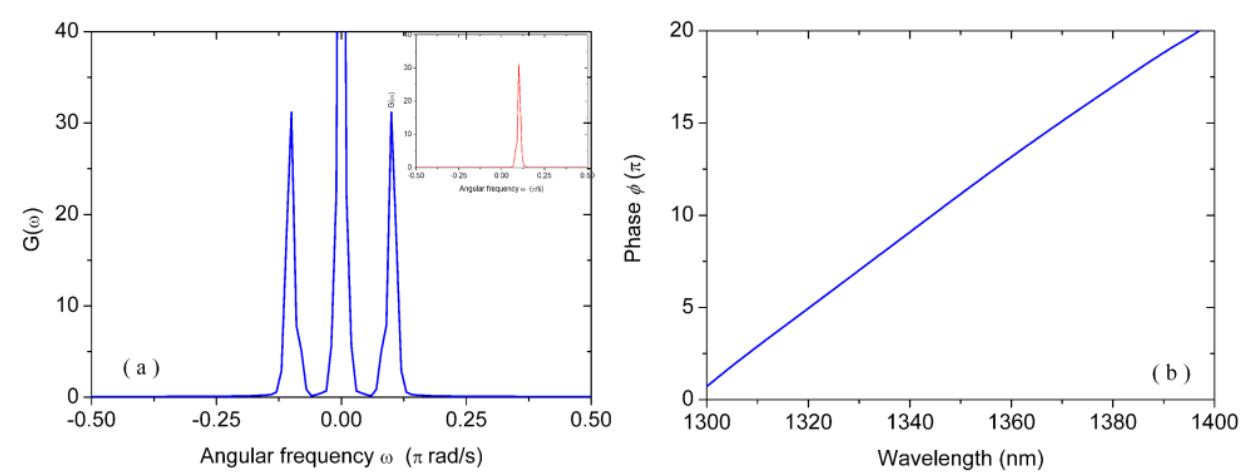

Figure 3. Fourier processing of fringe pattern: (a) Fourier spectrum (inset: filtered spectrum); (b)

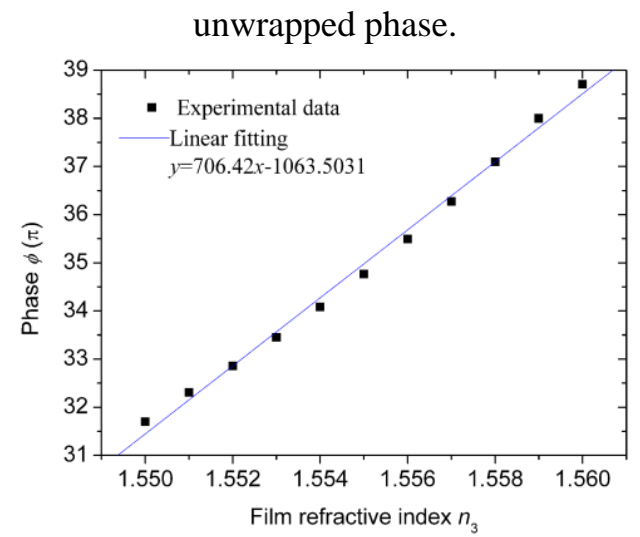

Figure 4. Dependence of the phase on film refractive index $(\lambda=1345.0 \mathrm{~nm})$. 


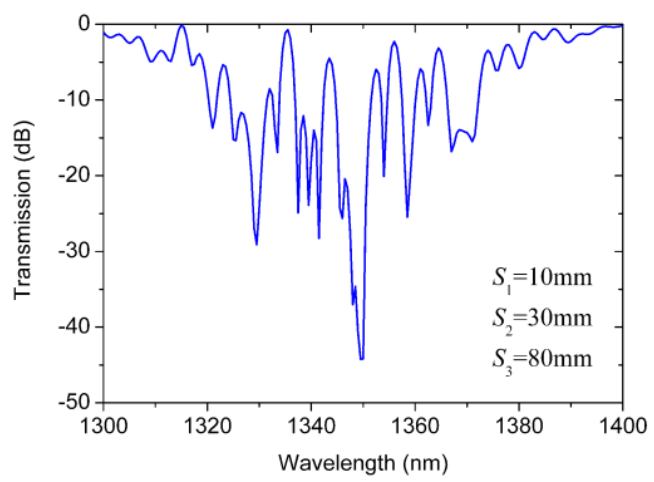

Figure 5. Composite transmission spectrum of three CLPFGs with different separations.
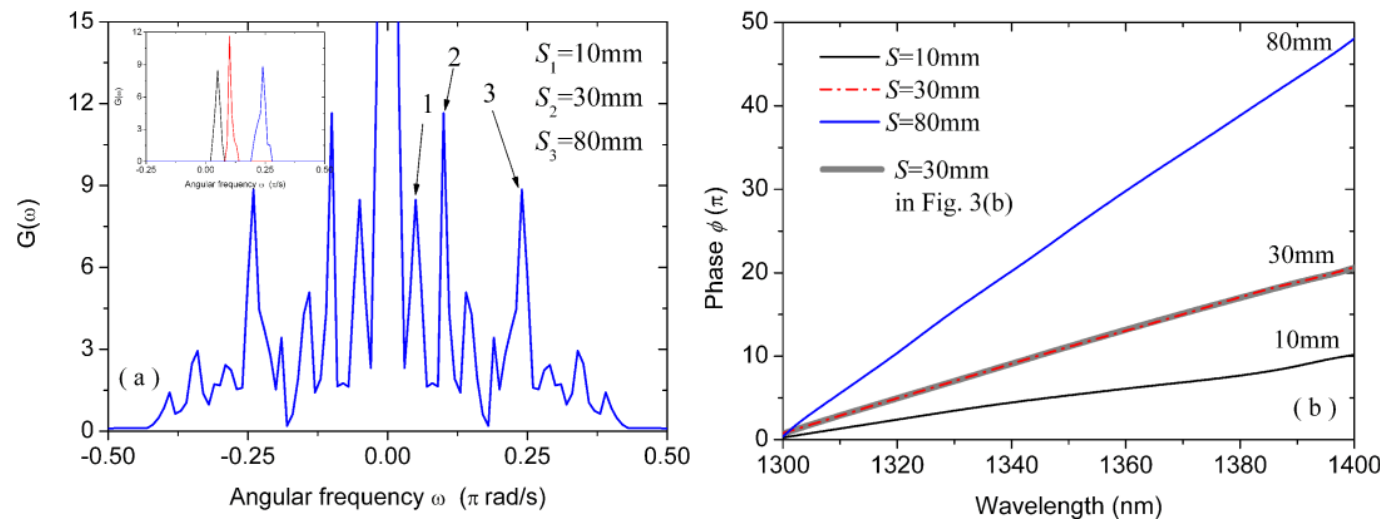

Figure 6. Fourier processing of composite fringe patterns: (a) Fourier spectrum (inset: three filtered characteristic peaks); (b) unwrapped phase.

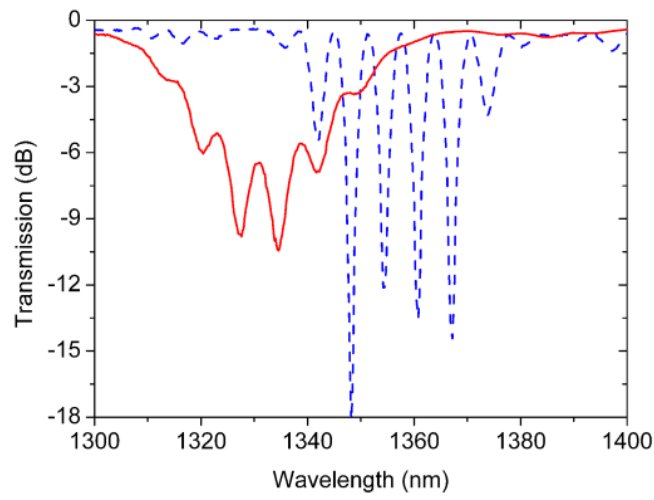

Figure 7. Transmission spectrum of $\mathrm{CLPFG}_{2}$ before (dashed line) and after (solid line) deposition of PAH/PAA film
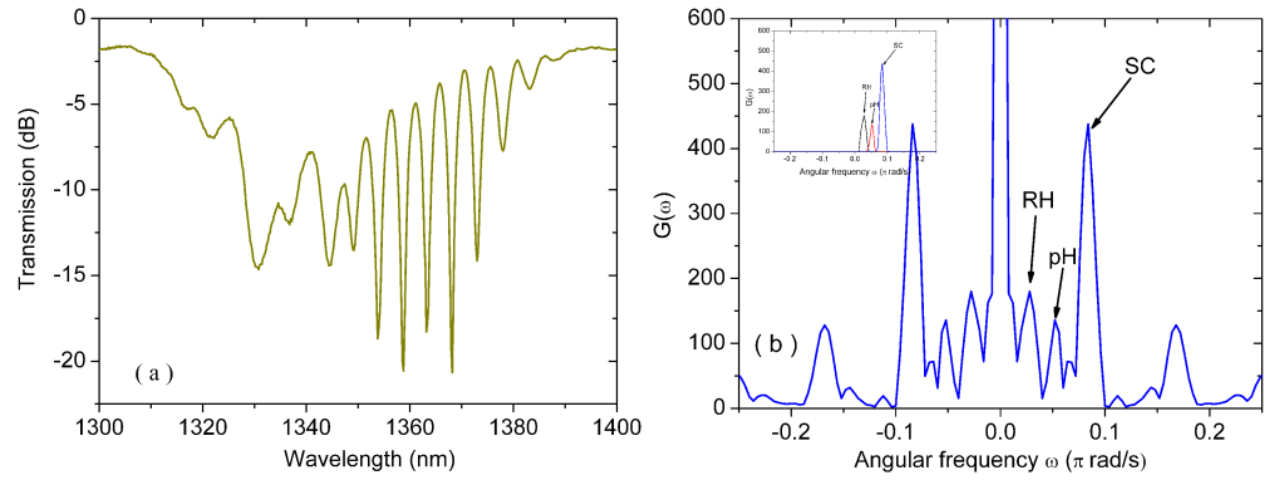


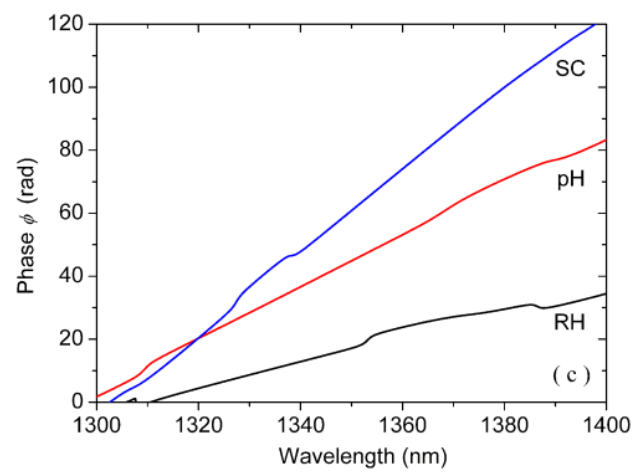

Figure 8. Fourier processing of measured composite spectrum $(\mathrm{RH}=53 \%, \mathrm{pH}=7, \mathrm{SC}=0 \%)$ : (a) composite transmission spectrum; (b) Fourier spectrum (inset: filtered spectra); (c) recovered phases.

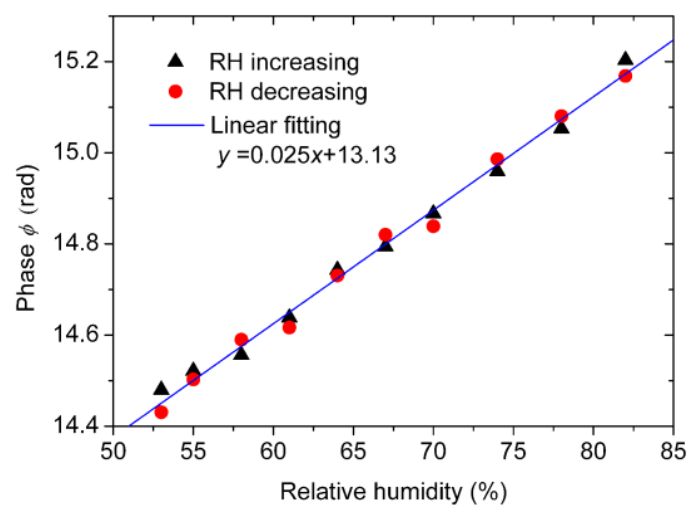

Figure 9. Response of $\mathrm{CLPFG}_{1}$ coated with $\mathrm{TiO}_{2} / \mathrm{SnO}_{2}$ film to $\mathrm{RH}(\lambda=1329.0 \mathrm{~nm}, \mathrm{pH}=7$, $\mathrm{SC}=0 \%)$.
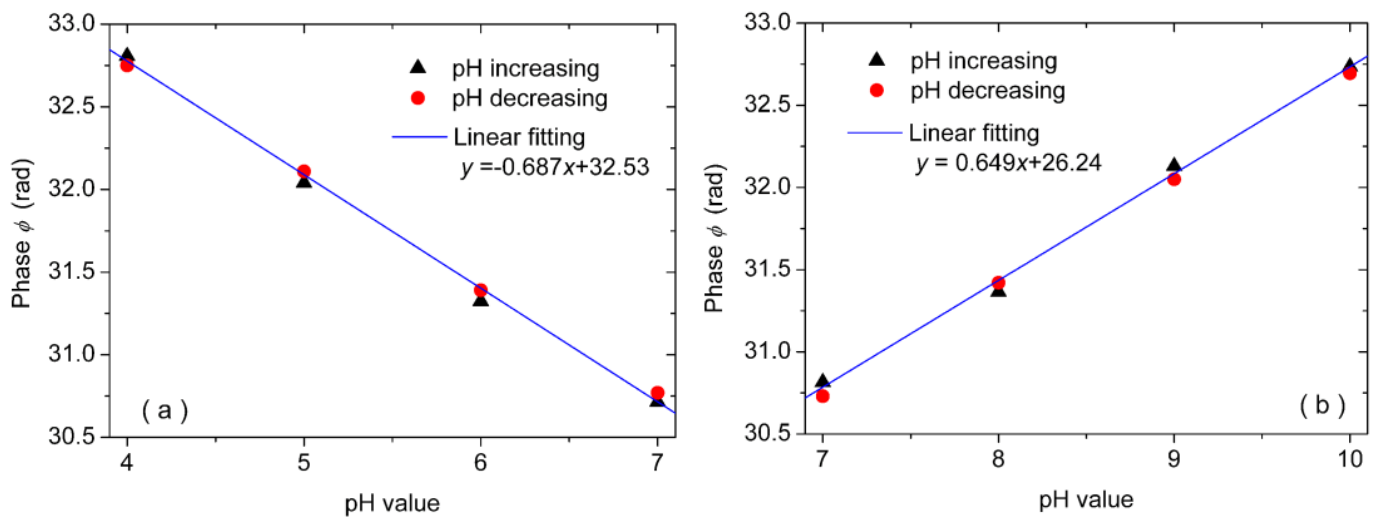

Figure 10. Response of $\mathrm{CLPFG}_{2}$ coated with $\mathrm{PAH} / \mathrm{PAA}$ film to $\mathrm{pH}$ value in (a) acidic and (b) alkaline solutions $(\lambda=1332.6 \mathrm{~nm}, \mathrm{RH}=53 \%, \mathrm{SC}=0 \%)$. 


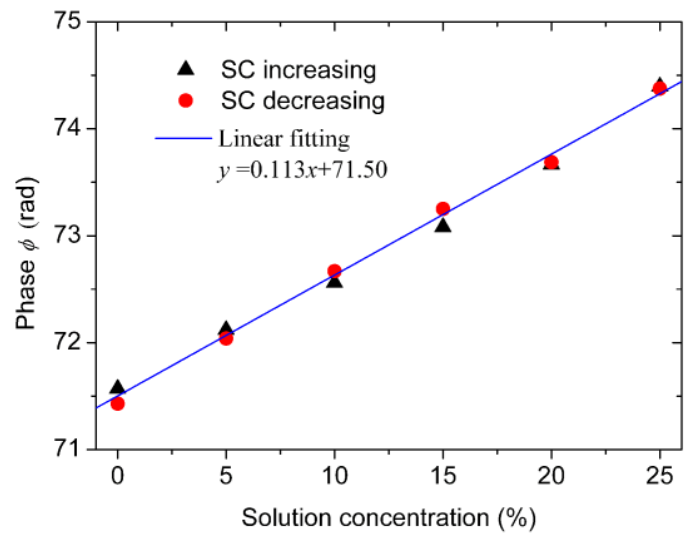

Figure 11. Response of $\mathrm{CLPFG}_{3}$ to $\mathrm{NaCl}$ solution concentration ( $\left.\lambda=1358.2 \mathrm{~nm}, \mathrm{RH}=53 \%, \mathrm{pH}=7\right)$.

Table 1. Different parameter combinations and the corresponding recovered phases

\begin{tabular}{cccccc}
\hline $\mathrm{RH}(\%)$ & $\mathrm{pH}$ & $\mathrm{SC}(\%)$ & $\phi_{\mathrm{RH}}(\mathrm{rad})$ & $\phi_{\mathrm{pH}}(\mathrm{rad})$ & $\phi_{\mathrm{SC}}(\mathrm{rad})$ \\
\hline 53 & 7 & 0 & 14.5436 & 30.5398 & 71.6710 \\
53 & 10 & 0 & 14.5432 & 40.9438 & 71.6712 \\
74 & 7 & 0 & 15.1646 & 30.5382 & 71.6711 \\
53 & 7 & 10 & 14.5430 & 30.5379 & 72.5925 \\
64 & 8 & 15 & 14.7456 & 31.0243 & 73.0982 \\
\hline
\end{tabular}

